\title{
Immobilization and Removal of Hydrocarbon Contamination Using the Evactron® De-Contaminator
}

\author{
Ronald Vane
}

XEI Scientific, 1755 E. Bayshore Rd. Suite 17, Redwood City, CA 94063

Background

The Evactron ${ }^{\circledR}$ De-Contaminator is well known for its ability to reduce and remove Hydrocarbon contamination artifacts in Electron Microscopes. The primary mechanism for this control is the oxidation of $\mathrm{HC}$ deposits to form short chain volatile compounds and oxides that can be pumped out the vacuum pumps. Comparison of RGA results on the removal of volatile components and visual observance of the removal of Hydrocarbon films indicates that the immobilization of Hydrocarbons on surfaces by Polymerization is also an important mechanism for reducing contamination interference with imaging in electron microscopy.

\section{Experimental}

XEI uses vacuum chamber equipped with a large window to study Hydrocarbon removal using the Evactron De-Contaminator. The Evactron De-contaminator uses RF plasma in a Oxygen Radical source connected to a chamber port to make $\mathrm{O}$ radicals from air, and then uses the pumping differential to flow the Oxygen radicals though the specimen chamber and oxidize the $\mathrm{HC}$ on all surfaces. Removal involves forming short chain molecules that can be volatilized and carried to the roughing pump such $\mathrm{CO}, \mathrm{CO} 2$, $\mathrm{H} 2 \mathrm{O}$, and short chain organic oxides such as alcohols, ketones, and esters. Polymerization involves cross linking the Hydrocarbon molecules under the influence of the oxidizing Oxygen radicals. The Oxygen radicals are most apt to attack by Hydride extraction, the removal of Hydrogen. If the resulting free radical site is not attacked quickly by another Oxygen radical, polymerization with adjacent molecules can occur. Polymerization creates longer chain molecules that less volatile and are more resistant to oxidation due to the higher energy of the Carbon - Carbon single bond. In the XEI chamber, we study the removal of Hydrocarbon deposits form mirrored surfaces because these results in an easily observed end point for cleaning. In the test chamber Evactron cleaning creates dark areas where there are thicker layers of Hydrocarbons and these dark deposits then are removed more slowly. These black areas are attributed to be oxidation induced polymers.

An Extorr XT 300 RGA was connected to the chamber with a Turbo pump pumping station so that the chamber could be pumped down low enough for the RGA to operate. The Extorr RGA was selected because it contains a pirani gauge as well as high vacuum ion gauge for total pressure measurements. It is designed to be tolerant of medium vacuum (10-4 Torr) operation and can be turned on early in the pump down process. The data shows that the black polymer deposits were not incompatible with clean RGA spectra. This finding shows that immobilization of Hydrocarbons by Evactron cleaning may be an important mechanism for preventing electron beam induced contamination in images.

Pump down times: The Evactron cleaning significantly improves pump down times on vacuum chamber exposed to air. On our test chamber the pump down time to turn on pressure for the RGA is dropped from over 10 minutes to less than two minutes after Evactron use. The accumulation of Water vapor on vacuum surfaces is the main contributor to long pump down times. The Evactron ORS plasma generates UV light which desorbs water vapor from the chamber walls. The water vapor is then efficiently carried 
to the pumps by the viscous flow transport mechanism. In addition we observed AMC hydrocarbon peaks in chambers left open to room air. Evactron cleaning during pump down was found to remove these AMC peaks from the RGA spectrum.

\section{Results}

Figures 1 and 2 show the changes in the RGA mass spectrum before and after Evactron Cleaning. The general background level baseline is significantly reduced in the post cleaning spectrum because of the ability of the Evactron to free gaseous species by both oxidative chemical etch and by UV activation. In Figure 1 there are hydrocarbon peak series showing between Mass 55 and 59 and another peak at 69 that are removed by Evactron cleaning in Figure 2. Additional data shows visible immobilized Hydrocarbon polymers on test mirrors without hydrocarbon peaks in the RGA spectrum. These results are compared with real world data taken on an SEM that correlates the low and high RGA partial presures are hydrocarbon with contamination deposits in images.

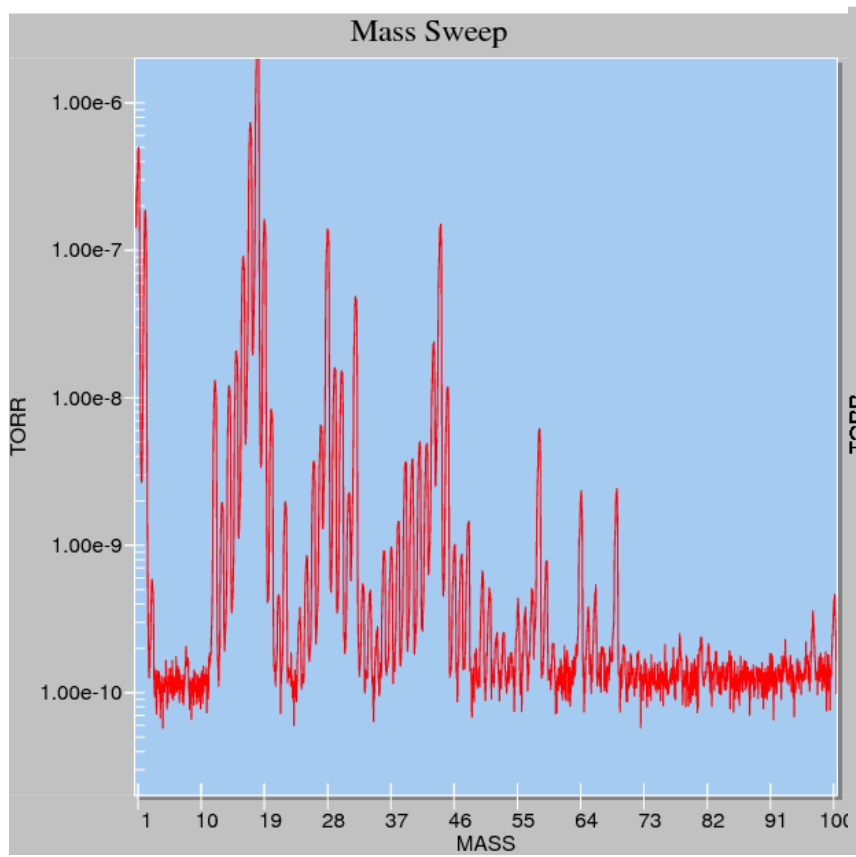

Figure 1

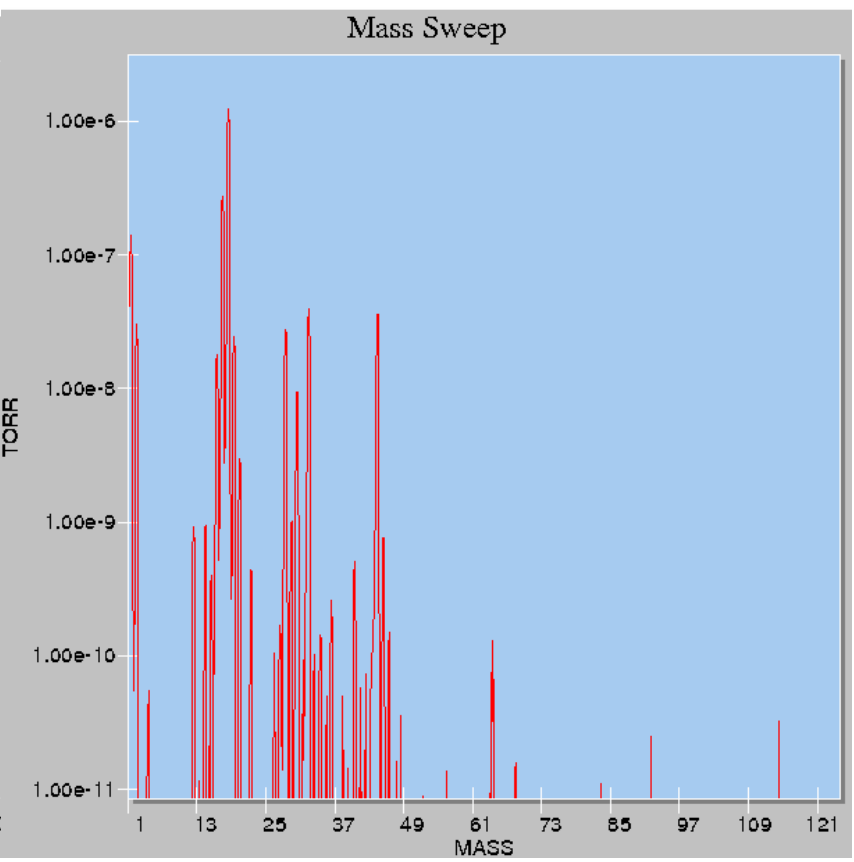

Figure 2

Figure 1 Mass spectra of test chamber before cleaning.

Figure 2 Mass Spectrum after Evactron cleaning

\section{Conclusions}

Evactron Cleaning reduces Contamination artifacts in electron microscopy by both oxidizing hydrocarbons and by inducing polymerization that reduces Hydrocaron vapor pressure. Vapor phase transport of Hydrocarbon to specimen surfaces and into the electron beam is probably an important mechanism for the spread of contamination in a chamber to the imaged area. Data shows that reducing vapor phase Hydrocarbons by the use of Evactron cleaning to remove it or to immobilize it improves imaging. 